WIENER SLAVISTISCHES JAHRBUCH, Band 57/2011, 159-164

(C) 2011 by Österreichische Akademie der Wissenschaften, Wien

Eva Maria O S S A D ÍK

\title{
Neue Denotate im kroatischen Zivilisationswortschatz: Die Revolution von 1848 und die Gesellschaft im Spiegel der zeitgenössischen Presse
}

Das Material für diese Arbeit stammt aus dem kroatischen Teil des Forschungsprojekts „'Gesellschaft' und 'Wirtschaft': Bosnisch, Kroatisch, Serbisch“, das von 2003 bis 2007 in der Balkan-Kommission der Österreichischen Akademie der Wissenschaften unter der Leitung von Herrn Prof. Dr. G. Neweklowsky bearbeitet wurde. Dieses Projekt ist die Fortsetzung des Forschungsprojekts „'Herrschaft' und 'Staat': Bosnisch, Kroatisch, Serbisch“, das Teil des Bündelprojekts „Der Zivilisationswortschatz im südosteuropäischen Raum 1840-1870“ ist und dessen Konzeption von Prof. Dr. R. Katičić stammt. Die Finanzierung beider Projekte erfolgte durch den „Fonds zur Förderung der wissenschaftlichen Forschung“.

Die Revolution in Wien wurde durch die Revolution in Paris am 23. Februar 1848 ausgelöst. Sie wirkte sich sofort auf die Presse aus, da die Zensur aufgehoben wurde. In Nummer 24 der Zeitung „Novine dalmat.-horvatsko-slavonske“ ('Dalmatinisch-kroatisch-slawonische Zeitung') vom 22. März 1848 erschien auf der ersten Seite in zwei Zentimeter großen Lettern die Meldung, dass die Presse frei sei („Štampa je slobodna“), und zwar - wie im Untertitel eines Artikels von Bogoslav Šulek zu lesen ist - seit dem 15. März um 1/2 6 Uhr nachmittags. Dieser Artikel umfasst eine Seite im Format A3.

Die Illyrische Bewegung (ilirski pokret) wollte eine gemeinsame Sprache der Südslawen bilden und das Kroatische von Fremdeinflüssen befreien; in ihrer Auswirkung auf die Neubildung von Wörtern des kroatischen Zivilisationswortschatzes in der ersten Hälfte des 19. Jahrhunderts ist sie bisher in relativ geringem Ausmaß bearbeitet worden ${ }^{1}$, da sie vor allem eine politische Bewegung war (vgl. Moguš 1995: 150).

${ }^{1}$ Vgl. Thomas 1988 mit einer Übersicht über die bisherige Literatur (S. 26-34). 
Mit der „Illyrischen Bewegung“, die sich in der Folge zur kroatischen nationalen Wiedergeburt entwickelte (vgl. Jonke 1975: 67), begann das kroatische Zeitungswesen (vgl. Katičić 2004: 19-27). Wie Auty bereits 1972: 51 hervorhebt, spielt das Ausmaß der Bereicherung der kroatischen Lexik durch $\mathrm{Lj}$. Gaj eine größere Rolle, als (zumindest bis dahin) erkannt worden ist; es ist aber äußerst schwierig, den namentlichen Urheber einer Neubildung festzustellen: Selbst wenn der Artikel, in dem sie vorkommt, namentlich gezeichnet sein sollte, ist kaum festzustellen, ob sie nicht in einer anderen Quelle oder in der zeitgenössischen mündlichen Sprache bereits seit längerem gebraucht worden ist.

Die Zeitung „Novine dalmat.-horvatsko-slavonske“ war 1835 von Ljudevit Gaj gegründet worden, der auch die Redaktion dieser Publikation übernahm und sie ebenso wie die auch von ihm gegründete Zeitschrift „Danica ilirska“ ('Der illyrische Morgenstern'), die sich aus einer literarischen Beilage zu dieser Zeitung entwickelte, als Plattform zur Verbreitung der Ideen des Illyrismus und durch die Sprachreform des Illyrismus bedingter Wörter (vgl. Despalatović 1975: 79) benützte. Der erwähnte Artikel ist jedoch von Bogoslav Šulek, seinem engen Mitarbeiter, gezeichnet, woraus man schließen könnte, dass er zu dieser Zeit selbst Redakteur war².

Wörter des sich im Ausbau befindlichen kroatischen Zivilisationswortschatzes, von denen sich der Redakteur nicht sicher war, ob sie auch verstanden werden würden (vgl. Katičić 2007: 306-307), sind in den kroatischen Zeitungen um 1848, „Narodne novine“ und „Slavenski Jug“, durch in Klammern nachgestellte Kontaktsynonyme erklärt - bei solchen Beispielen kann man sicher sein, dass es sich um (alte) Wörter mit neuen Bedeutungen handelt. Solche Wörter, die die kroatische Gesellschaft betreffen und 1848 in diesen beiden Zeitungen belegt sind, wollen wir hier erörtern.

'Presse' gehört zu den Denotaten, deren Benennung zur Zeit der Revolution von 1848 erst im Entstehen war: 1847 und 1848 sind in unserem Material sowohl štampa als auch tisak belegt: Štampa aus ital. stampa mit deutscher Aussprache des anlautenden st- (z. B.: N 80/1847, 319/1/453; DI 44/1848, 177/2/9 und SJ 11/1848, 44/1/30) war offensichtlich der übliche Ausdruck, da er nie durch ein Kontaktsynonym glossiert wurde. 1847 ist aber auch erstmals tisak belegt, das seinen Gebrauch für 'Presse' der Illyristischen Bewegung verdankt: Es sollte das fremdstämmige štampa ersetzen. In jedem Beleg ist es in Klammern durch ein Kontaktsynonym glossiert: tisak (stampa) (N 23, 91/1/42 [1] $]^{4}$ ) und tisak (Presse) (N 58, 230/2/11 [2]); tisak bedeutete etymologisch gesehen 'Druck'. 1848 ist tisak (SJ9, 35/2/22 [6]) ohne

${ }^{2}$ Es ist zwar bekannt, dass er eine Zeit lang Redakteur der Zeitung ,Novine dalmat.-horvatsko-slavonske“" war, die genaue Zeit dieser Tätigkeit ist jedoch nicht belegt.

3 Angaben von Zitaten aus Zeitungen sind wie folgt zu verstehen: N 80/1847, 319/1/45 = Narodne novine (Kurzfassung des Titels „Novine dalmat.-horvatsko-slavonske“), Nummer 80 des Jahres 1847, Seite 319, 1. Kolumne, Zeile 45.

${ }^{4}$ Ziffern in eckigen Klammern beziehen sich auf die Beispiele am Ende dieses Aufsatzes. 
nachfolgende erklärende Glossierung belegt. Heute hat sich tisak durchgesetzt, štampa ist umgangssprachlich (und serbisch).

Das Denotat 'Pressefreiheit' wurde entweder mit sloboda tiska (der früheste Beleg aus „Narodne novine“ stammt aus dem Jahr 1847 und beschreibt die Situation in Deutschland, die Belege aus dem Jahr 1848 sowohl in „Narodne novine“ als auch in „Slavenski Jug“ beziehen sich thematisch auf Kroatien), in mehreren Belegen durch sloboda štampe und einmal durch sloboda pečatnje (SJ 32/1848, 127/1/50) - pečatnja ist aus dem russischen печатня 'Druckerei' entlehnt (ARj. s. v.) und im Kroatischen nicht erhalten geblieben - ausgedrückt. Sie werden aber nicht durch ein Kontaktsynonym erklärt.

Eine konkrete Grundlage für dieses Denotat gab es bald nicht mehr: Die „oktroyierte" Verfassung vom 4. März 1849 machte die Pressefreiheit wieder rückgängig (vgl. Bauer 1942: 38).

Auch für ein anderes neues Denotat im Zusammenhang mit der Presse, nämlich 'Pressegesetz', gab es mehrere Ausdrücke: zakone tiska (Pressgesetze) [4], zakon o pečatnji (SJ 32/1848, 127/1/5) und štamparski zakon (SJ 59/1848, 233/2/6) - die entsprechenden Wortgruppen in ,Slavenski Jug“ wurden nicht mehr mit einem folgenden erklärenden Klammerausdruck versehen.

Die Revolution von 1848 hatte neben politischen vor allem gesellschaftliche Auswirkungen: Die Gesellschaft, also die Gesamtheit der Bürger in einem liberalen Staat, musste jetzt benannt werden, da des öfteren von ihr die Rede war. Das Wort društvo, das bis dahin ausschließlich 'Gesellschaft, Verein' ${ }^{5}$ bedeutet hatte, wurde erstmals in „Slavenski Jug“6 Nr. 1 vom 6. August 1848 in der neuen Bedeutung verwendet: Nach Ansicht der Redakteure war es notwendig, dieses Wort mit dem Adjektiv čovječanski 'menschlich' zu versehen [5] oder mit frz. société7 zu glossieren [6], damit es auch verstanden wurde. Das deutsche Wort Gesellschaft eignete sich als Kontaktsynonym nicht, da es genau so zweideutig ist wie kroat. društvo. Das Adjektiv društven ist in der Bedeutung 'gesellschaftlich' in „Narodne novine“ 1847 häufig belegt - meist in den Wendungen 'Gesellschaftsleben' oder 'gesellschaftliche Verbindungen', auch in der Verbindung 'Gesellschaftsordnung', hier allerdings auf Russland bezogen.

Auch der Staat musste genau benannt werden, nämlich država, ursprünglich 'Land', 'Gegend', 'Region', 'Besitz', 'Landbesitz', 'Ordensprovinz', 'Bistum',

5 Vgl. Katičić 2001: 170.

${ }^{6}$ Dieses Blatt konnte nach Bauer 1942: 37 ,jeden Vergleich mit einer der damaligen großen europäischen Zeitungen vertragen."

7 Société kann auch im Frz. die Bedeutung 'Verein' haben, die Bedeutung 'die Gesellschaft' ist aber häufiger. - Die in diesem Aufsatz zitierten Beispiele aus „Slavenski Jug“, die in einem nachfolgenden Klammerausdruck mit einem französischen Kontaktsynonym versehen sind, stammen aus einem namentlich nicht gezeichneten Artikel mit dem Titel „Pogled na europejske dogadjaje god. 1848“ 'Blick auf die europäischen Geschehnisse des Jahres 1848'. 
'Pfarre, ${ }^{8}$ mit der erklärenden Glosse l'état [6] - der Staat war nach der Revolution nicht mehr derselbe wie davor.

Ein Wort für den Staatsbürger gab es schon früher - državljanin -, in Nummer 19 von „Narodne novine“ vom 4. März 1848 ist državljanstvo [7] mit dem Kontaktsynonym Staatsbürgerschaft belegt, das vorläufig den Erstbeleg dieses Wortes darstellt: Dafür spricht außer seiner Glossierung, dass es laut ARj. erst bei M. Pavlinović $1876^{9}$ vorkommt. In Nummer 26 von „Slavenski Jug“ vom 5. Oktober 1848 $(104 / 1 / 63)$ ist dieses Wort bereits ohne erklärende Glosse belegt.

Der Staat musste rechtlich in einer Verfassung festgelegt werden: Das fremdstämmige Wort konstitucia/konštitucia wurde durch das kroatische ustav in den Jahren 1847 und 1848 ersetzt, ustav wurde aber im oben erwähnten Aufsatz mit dem Titel „Štampa je slobodna“ durch in Klammern folgendes konstitucia erklärt. So ist auch verständlich, dass neustavnost in der ersten Nummer von "Slavenski Jug“ durch inconstitutionalité [8] glossiert wird.

Ein Wort für 'Recht' (pravo) gab es schon seit urslawischer Zeit, jetzt wurde aber ein neues Konzept erdacht, nämlich das 'Menschenrecht', ein Recht, das jedem Menschen ohne Ansehen der Person zusteht. Dieser neue Begriff musste ins Kroatische übersetzt und auch verstanden werden können: In der 1. Nummer von „Slavenski Jug" (2/1/14) wurde er mit prava čovjeka übersetzt, dem in Klammern das erklärende „droits de l'homme“ folgt.

Zusammenfassend kann man feststellen, dass anhand weniger repräsentativer Beispiele gezeigt werden kann, wie der Ausbau der kroatischen Schriftsprache um die Mitte des 19. Jahrhunderts funktionierte:

- Wörter aus fremden Sprachen wurden - hier macht sich die Illyrische Bewegung bemerkbar - durch kroatische ersetzt: štampa durch tisak; der Ersatz durch das immerhin slawische russ. печатня, das eine etwas andere Bedeutung ('Druckerei') hat, hat sich nicht durchgesetzt. Heute heißt 'Presse' tisak, štampa ist umgangssprachlich. In ähnlicher Weise wurde konštitucia/konstitucia durch ustav ersetzt, ustav hat sich durchgesetzt.

- Neue Denotate wurden zunächst durch mehrere Wörter bzw. Wortfügungen bezeichnet, von denen sich mit der Zeit eine(s) durchsetzt: Für 'Pressefreiheit' gab es sloboda tiska, sloboda štampe und sloboda pečatnje, heute wird dieses Denotat mit sloboda tiska bezeichnet. Das 'Pressegesetz' wurde mit zakon tiska, zakon štamparski und zakon o pečatnji bezeichnet, heute heißt es zakon o tisku.

- Die Wörter društvo 'Gesellschaft' und država 'Staat' erhielten eine neue Bedeutung, denn Gesellschaft und Staat sahen nach der Revolution von 1848 anders aus als vorher.

${ }^{8}$ Die in unserem Material vorhandenen Belege für 'Staat' lauten alle država. - Zur Etymologie dieses Wortes s. Katičić 2001: 170.

9 Razgovor o slavenstvu, jugoslavenstvu, srbo-hrvatstvu, Zadar ${ }^{3} 1876$. 
- Das Wort für 'Staatsbürger' državljanin war eine Neubildung, nämlich eine Ableitung mit dem kroat. Suffix -janin von dem bereits vorhandenen Substantiv $d r$ žava. Ebenso wurde der neue Begriff des Menschenrechts mit kroatischen Mitteln (prava čovjeka) benannt.

\section{Liste der erwähnten Beispiele}

Im Gegensatz zum Aufsatz wird in den zitierten Beispielen die Originalorthographie beibehalten.

[1] Govori se, da papa naměrava tisak (štampu) oslobodit od sadašnjega ugnjetavanja zavedenjem u Rimu vèrhovnoga veća za cenzuru. 'Man spricht darüber, dass der Papst beabsichtigt, die Presse von der jetzigen Unterdrückung des in Rom eingerichteten Zensurrats zu befreien.' (N 23/1847, 91/1/42)

[2] Kao jamstvo ustavnoga života u Ugarskoj, (...), [oppozicia] smatra nadalje javnost političkoga života, tako u tisku (Presse), ... 'Als Garantie für ein verfassungsgemäßes Leben in Ungarn sieht die Opposition weiters die Öffentlichkeit des politischen Lebens, so auch in der Presse, an ...' (N 58/1847, 230/2/11)

[3] Uz knjigu bit će priložena jedna litografirana tablica kirilice, upotrěbljavane od r〈imo〉-katoličkih Jugoslavenah u pečatnji mletačkoj, ... 'Dem Buch wird eine lithographierte Tabelle des kyrillischen Alphabets, wie es von den römisch-katholischen Südslawen in der Druckerei von Venedig verwendet wird, beigelegt ...' (N 28/1847, 112/1/43)

[4] ..., neka se naimenuje odbor, koi će zakone tiska (Pressgesetze) izraditi; ... '..., damit ein Ausschuss ernannt werde, der Pressegesetze ausarbeiten soll.' (N 94/1847, 377/2/60)

[5] Dèržava i družtvo čoviečansko (...) su (...) sriedotočje najnoviega gibanja europejskog, kojemu kako je već rečeno, povod je dala Francia. 'Staat und Gesellschaft der Menschen sind der Mittelpunkt der neuesten europäischen Bewegung, für die - wie schon gesagt wurde - Frankreich der Anlass war.' (SJ 1/1848, 1/3/6)

[6] Tko ne zna dalje da u svemu onom, što se razvitka dèržave (l'état) tiče, narod francezki od davnih vremenah predvodi narode europejske, i da u družtvo (la société) čoviečansko najdublje utica? 'Wer weiß weiters nicht, dass in allem, was die Entwicklung des Staates betrifft, das französische Volk seit alten Zeiten die europäischen Völker anführt und dass es die menschliche Gesellschaft am stärksten beeinflusst?' (SJ 1/1848, 1/3/2-4)

[7] Dèržavljanstvo (Staatsbürgerschaft) [u Napulju] dobiva se i gubi zakonom. 'Die Staatsbürgerschaft bekommt und verliert man [in Neapel] durch Gesetz.' (N1848/19, 77/1/80)

[8] Ono je nezakonitost, neustavnost (inconstitutionalité), slabost, ono je izdajstvo. 'Das ist Ungesetzlichkeit, Verfassungswidrigkeit, eine Schwäche, das ist Verrat.' (SJ 11/1848, $1 \mathrm{a} / 1 / 41)$

[9] Pisma revolucie puna su razlogah o pravih čovieka (droits de l'homme) ... 'Die Revolutionsschriften sind voll von Gründen für Menschenrechte ...' (SJ 1/1848, 2/1/14)

$\mathrm{N}=$ Novine narodne

$\mathrm{SJ}=$ Slavenski Jug (für das Material aus dieser Zeitung bedanken wir uns bei Frau Dr. Nevenka Petković)

\section{Literatur}

ARj. 1884-1975: $\quad$ Rječnik hrvatskoga ili srpskoga jezika, Zagreb 1884-1975

Auty 1972:

Robert Auty, Sources and Methods of Lexical enrichment in the Slavonic Language - Revivals of the Early Nineteenth century, in: D. S. Worth, The Slavic Word: proceedings of the International Slavistic Colloquium at UCLA, The Hague, 41-52 
Bauer 1942:

Ernest Bauer, Die Entwicklung der Publizistik in Kroatien, Zagreb

Despalatović 1975: Elinor Murray Despalatović, Ljudevit Gaj and the Illyrian Movement, East European Monographs 12, New York - London

Hansen-Kokoruš 2005: R. Hansen-Kokoruš, J. Matešić, Z. Pečur-Medinger, M. Znika, Deutsch-kroatisches Universalwörterbuch, Red. J. Matešić und D. Brozović Rončević, Zagreb

Jonke 1975: Ljudevit Jonke, Ljudevit Gaj kao višestruki pobjednik, Jezik 23, 6779

Katičić 2001: $\quad$ Radoslav Katičić, Die Revolution 1848 im Spiegel des auf Herrschaft und Staat bezogenen Wortschatzes der zeitgenössischen kroatischen Publizistik, Anzeiger der philosophisch-historischen Klasse der Österreichischen Akademie der Wissenschaften 136, 169-175

Katičić 2004: $\quad$ Radoslav Katičić, Der auf Politisches bezogene Wortschatz der kroatischen Illyristen in den frühen vierziger Jahren des 19. Jahrhunderts, in: Gerhard Neweklowsky (Hrsg.), Herrschaft, Staat und Gesellschaft in Südosteuropa aus sprach- und kulturhistorischer Sicht. Erneuerung des Zivilisationswortschatzes im 19. Jahrhundert (= Österreichische Akademie der Wissenschaften, Schriften der Balkan-Kommission 48), Wien, 29-72

Katičić 2007: $\quad$ Radoslav Katičić, Ergebnisse des Symposions und Schlusswort, in: G. Neweklowsky (Hrsg.), Herrschaft, Staat und Gesellschaft in Südosteuropa aus sprach- und kulturhistorischer Sicht, Erneuerung des Zivilisationswortschatzes im 19. Jahrhundert. Akten des Internationalen Symposiums 2.-3. März 2006, Wien 2007, 303-308

Moguš 1995: Milan Moguš, Povijest hrvatskoga književnoga jezika, Zagreb

Thomas 1988:

George Thomas, The Impact of the Illyrian Movement on the Croatian Lexicon (= Slavistische Beiträge 223), München

A b stract: Newly formed words in the Croatian vocabulary concerning civilization. The Revolution of 1848 and society as reflected in the contemporary newspapers. The revolution of 1848 caused the coining of new words concerning society, which had undergone a considerable change. The Illyrian Movement, the Croatian form of national awakening, strongly influenced this process: new words were preferably built of Croatian or at least Slavic elements and sometimes replaced older ones of foreign origin.

K e y w or d s : language, beginning of the 19th century, national elements, vocabulary of civilization, new words

Eva Maria Ossadník

Österreichische Akademie der Wissenschaften

Balkan-Kommission,

Fleischmarkt 22, 1010 Wien, Österreich

eva-maria.ossadnik@oeaw.ac.at 